ISSN 1991-8631

Original Paper

http://indexmedicus.afro.who.int

\title{
Diversité phytoplanctonique et niveau de pollution des eaux du collecteur de Dantokpa (Cotonou-Bénin)
}

\author{
Dogbè Clément ADJAHOUINOU ${ }^{1 *}$, Nourou Dine LIADY ${ }^{1,2}$ et Emile Didier FIOGBE ${ }^{1}$ \\ ${ }^{I}$ Unité de Recherche sur les Zones Humides, Département de Zoologie, Faculté des Sciences et Techniques, \\ Université d'Abomey-Calavi, 01BP526 Cotonou, Bénin ; Tél : 0022921300074 (Poste 166). \\ ${ }^{2}$ Unité «Assainissement et Environnement», Département Sciences et Gestion de l'Environnement, Université \\ de Liège, Avenue de Longwy 185, B - 6700 ARLON, Belgique; \\ Tél. : 003263230849 - Fax : 003263230800. \\ *Auteur correspondant; E-mail : clementiofr@yahoo.fr, Tél : 22997265322
}

\section{RESUME}

La présente étude a été conduite pour apprécier le niveau de pollution des eaux du collecteur de Dantokpa (Cotonou-Bénin) par l'évaluation de la diversité phytoplanctonique en relation avec les caractéristiques physico-chimiques afin de faire ressortir le risque de leur rejet sans traitement dans la lagune de Cotonou. L'analyse des eaux usées prélevées au niveau de trois stations a révélé des teneurs élevées en orthophosphates et en minéraux azotés (ammonium, nitrate et nitrite), une forte conductivité électrique et un faible taux d'oxygène dissous. Au total, 39 espèces phytoplanctoniques ont été identifiées au microscope et sur la base des clés d'identification. Cette communauté hétérogène de phytoplancton se répartit dans 5 embranchements (Chlorophytes, Chrysophytes, Cyanophytes, Euglénophytes et Rhodophytes) et 9 classes dominées par les Cyanophycées et les Diatomophycées. La densité phytoplanctonique a été élevée avec une moyenne de 25760 cellules/ml. Les paramètres étudiés témoignent de l'abondance de l'azote, du phosphore et des matières organiques dans les eaux du collecteur. Il est donc nécessaire de les épurer avant leur déversement dans la lagune au risque de provoquer son eutrophisation.

() 2012 International Formulae Group. All rights reserved.

Mots clés: phytoplancton, eaux usées, épuration, eutrophisation, Cotonou.

\section{INTRODUCTION}

Dans la majorité des pays en développement, plus de $90 \%$ des eaux usées sont déversées directement dans la nature sans avoir subi de traitement selon le rapport du Programme des Nations Unis pour l'Environnement (PNUE) pour l'année 2006. Ceci est dû à l'inexistence et/ou à la défectuosité des systèmes de collecte et de traitement de ces eaux dans ces pays (Morel et al., 1998).
En Afrique de l'ouest, les systèmes classiques d'égout lorsqu'ils existent ne desservent que les centres-villes. Dans les quartiers périurbains, les eaux usées, à défaut d'ouvrages d'assainissement individuel, sont déversées dans les rues ou le plus souvent dans les caniveaux d'eaux pluviales (Koné, 2002). A Cotonou, métropole économique de la République du Bénin, cette situation est très préoccupante. En effet, dans cette ville, il n'existe ni égout spécifique aux eaux usées, ni 
station d'épuration. Une grande partie des eaux usées est déversée dans les collecteurs d'eaux pluviales et rejetée directement dans le lac Nokoué ou la lagune de Cotonou (MMEE, 2007). Ces pratiques intensifient ainsi graduellement la pollution chimique, organique et microbiologique de ces exutoires (Dovonou, 2008). Il est donc nécessaire d'envisager l'épuration de ces effluents avant leur rejet dans le milieu naturel.

Les méthodes intensives de traitement de ces eaux s'étant révélées inadaptées aux contextes africains en raison essentiellement de leur caractère onéreux (UADE et OIEAU, 1993), plusieurs auteurs ont proposé les procédés de type extensif notamment le lagunage, plus simple à exécuter, facile à entretenir et moins couteux (Seidl et Mouchel, 2003 ; Effebi, 2009). Le lagunage à microphytes, le plus connu des procédés de dépollution par voie naturelle (Koné, 2002), repose sur la capacité du phytoplancton à extraire certains polluants tels l'azote, les phosphates et les métaux lourds (Zerhouni et al., 2004) et à produire de l'oxygène grâce à la photosynthèse. Cette technique facilite ainsi la dégradation des matières organiques par les bactéries aérobies sans apport extérieur d'oxygène. Par ailleurs, en raison des propriétés bioindicatrices de la qualité des eaux qu'ont certaines espèces de phytoplancton (Iltis, 1980), ces organismes ont été proposés par la Directive Cadre de l'Eau (DCE) comme éléments de qualité biologique des écosystèmes aquatiques. Une étude des communautés phytoplanctoniques des eaux usées s'avère donc nécessaire pour mieux les caractériser en vue d'en assurer une épuration efficiente le cas échéant.

Le présent travail vise donc à apprécier le niveau de pollution des eaux du collecteur de Dantokpa (Cotonou-Bénin) par l'évaluation de la diversité phytoplanctonique en relation avec des paramètres physico-chimiques afin de faire ressortir le risque de leur rejet sans traitement dans la lagune de Cotonou.

\section{MATERIEL ET METHODES}

Présentation du milieu d'étude

Cette étude a porté sur les eaux du collecteur d'eau à ciel ouvert de Dantokpa.
Long d'environ $2,5 \mathrm{~km}$, cet ouvrage est située à Cotonou, entre $06^{\circ} 21^{\prime} 55.2^{\prime \prime}$ ' et $06^{\circ} 22^{\prime} 33.4^{\prime \prime}$, latitude Nord et $02^{\circ} 25^{\prime} 18.2^{\prime \prime}$ ' et $02^{\circ} 26^{\prime} 06.3^{\prime \prime}$, longitude Est. Il se dresse au cœur du marché Dantokpa sur environ $1 \mathrm{Km}$ et déverse son contenu dans la lagune de Cotonou qui relie le lac Nokoué à l'Océan Atlantique.

La zone d'étude en raison de sa situation géographique est soumise à un climat subéquatorial caractérisé par deux saisons pluvieuses (une grande d'avril à juillet et une petite marquée par la crue des eaux continentales d'octobre à novembre) et deux saisons sèches (une grande de décembre à mars et une petite couvrant les mois d'août et de septembre) (Adam et Boko, 1993). L'échantillonnage de l'eau a été effectué entre le 16 janvier et le 5 février 2011, période se situant dans la grande saison sèche où le canal ne reçoit que d'eaux résiduaires.

Considérant l'importance de sa longueur et en fonction de la pression anthropique croissante dont il fait l'objet de l'amont vers l'aval, trois stations ont été retenues le long du canal en vue d'apprécier les variations biotiques et abiotiques (Figure1). Ainsi, les Stations 1 et 3 sont respectivement situées en amont et en aval (à l'entrée de l'exutoire) du canal, la Station 2 située à l'entrée du marché.

\section{Echantillonnage et techniques d'analyse \\ $\mathrm{Au}$ total, quatre séances}

d'échantillonnage ont été effectuées avec une périodicité hebdomadaire et aux environs de la mi-journée où la transparence à la lumière est maximale, pour maximiser la récolte du phytoplancton (Findlay et Kling, 2003). A chaque station, l'eau a été prélevée au centre du collecteur, à l'aide d'une puisette dans des bouteilles en plastique de 1 L. Les échantillons devant servir à l'évaluation de la diversité phytoplanctonique sont fixés sur le terrain avec $5 \mathrm{~mL}$ d'une solution alcaline de lugol de commerce à la concentration finale de $0,5 \%$. Tous les échantillons ont été maintenus au froid dans une glacière pendant le transport, et immédiatement mis au réfrigérateur à $4{ }^{\circ} \mathrm{C}$ dès le retour au laboratoire. 
Juste avant les prélèvements d'eau, certains paramètres sont mesurés in situ. Il s'agit du taux d'oxygène dissous mesuré à l'aide d'un oxymètre portable WTW oxi 340i/SET, de la température, du pH et de la conductivité électrique (CE) mesurés avec un conductimètre portable multifonction WTW pH/cond 340i/SET.

$\mathrm{Au}$ Laboratoire d'Hydrologie Appliquée (LHA) de la Faculté des Sciences et Techniques (FAST), la turbidité et les matières en suspension (MES) ont été déterminées par colorimétrie (Hach, 2004) avec le colorimètre Hach DR/890; les sels nutritifs tels que les nitrates $\left(\mathrm{NO}_{3}{ }^{-}\right)$, les nitrites $\left(\mathrm{NO}_{2}{ }^{-}\right)$, l'ammonium $\left(\mathrm{NH}_{4}^{+}\right)$et les orthophosphates $\left(\mathrm{PO}_{4}{ }^{3-}\right)$ ont été dosés avec le chromatographe ionique de marque DIONEX ICS-1000.

$\mathrm{Au}$ Laboratoire d'Ecologie de l'Eau (LEE) de l'Unité de Recherche sur les Zones Humides (URZH), les espèces de phytoplancton ont été observées au microscope photonique de marque VWR.
Leur identification a été faite à partir des dessins et photographies réalisés aux objectifs $\times 40, \times 100$ (à immersion) et sur la base des clés d'identification de Bourrelly (1981, 1985, 1990), de Compère (1974, 1975a, 1975b, 1976a, 1976b, 1977) et d'Iltis (1980). La densité du phytoplancton a été estimée par dénombrement à l'aide d'une cellule de comptage (hématimètre) de type Burker (Helm et al., 2006).

\section{Analyses statistiques}

Le test d'analyse de la variance suivi du test d'homogénéité de Student-NewmanKeuls réalisé avec le logiciel SAS 9.2., ont été effectués après vérification des conditions de normalité et d'homoscédasticité de la variance, pour comparer les moyennes des données physico-chimiques et de la densité phytoplanctonique des stations. Par ailleurs, le test de comparaison de proportion réalisé avec le logiciel Minitab 14 a permis de comparer la diversité spécifique en phytoplancton des stations entre elles.

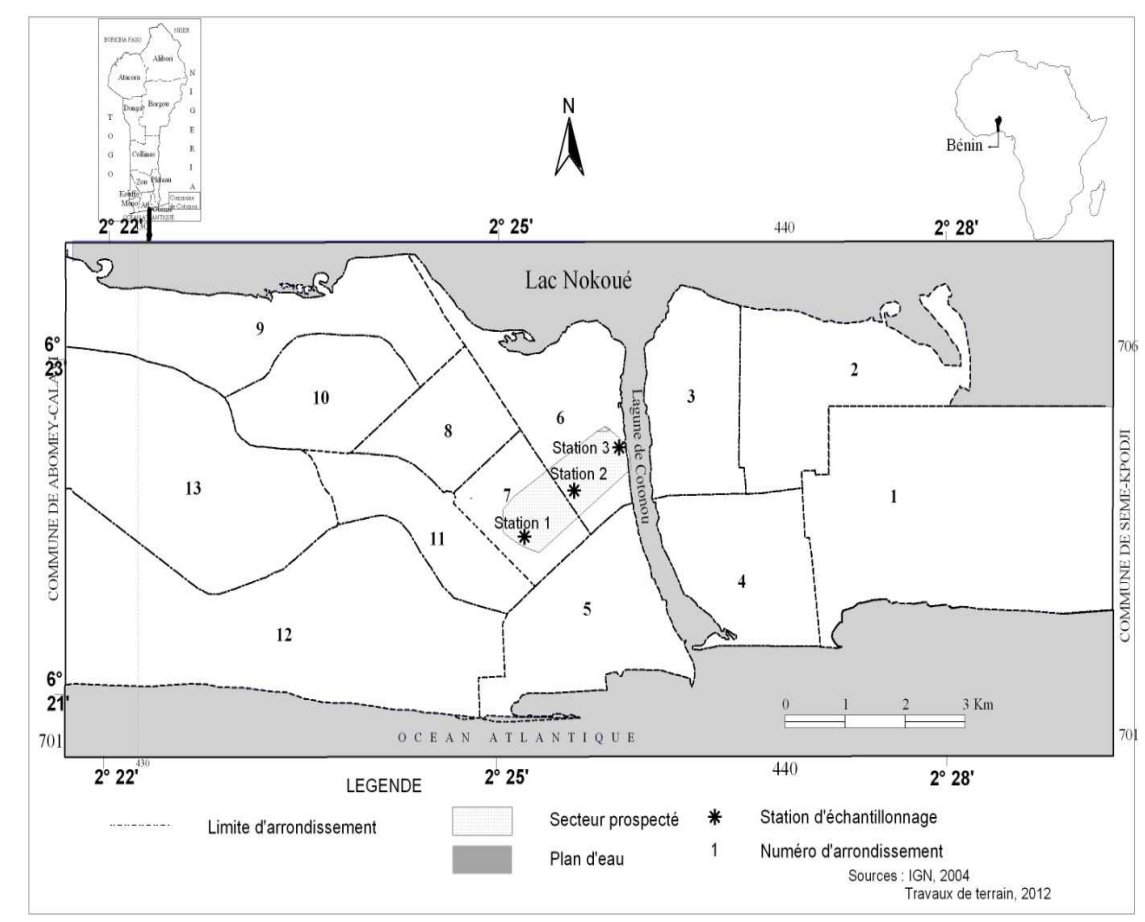

Figure1: Carte de la ville de Cotonou montrant les stations d'échantillonnage. 


\section{RESULTATS}

\section{Paramètres physico-chimiques}

Les paramètres physico-chimiques tels que la température, le $\mathrm{pH}$, le taux d'oxygène dissous, la turbidité, les matières en suspension, les nitrates, les nitrites, l'ammonium et les orthophosphates ont peu varié d'une station à une autre $(\mathrm{p}>0,05)$. Seule la conductivité électrique a significativement varié de $0,5 \mathrm{~ms} / \mathrm{cm}$ (Station 1) à $4,57 \mathrm{~ms} / \mathrm{cm}$ (Station 3) avec une moyenne plus élevée à la Station 3 qu'au niveau des deux autres stations (Tableau 1).

\section{Phytoplancton}

\section{Diversité taxonomique}

$\mathrm{Au}$ total, 39 espèces phytoplanctoniques ont été identifiées dans les eaux du canal (Tableau 2). Ces espèces appartiennent à 30 genres, 22 familles, 15 ordres, 9 classes et 5 embranchements à savoir Chlorophytes, Chrysophytes, Cyanophytes, Euglénophytes et Rhodophytes. L'embranchement des Chrysophytes est le plus représenté avec 15 espèces soit 38\% du peuplement (Figure 2). Les Cyanophycées et les Diatomophycées sont, de même, les classes les plus rencontrées avec chacune 12 espèces (Figure 3).

Le genre Oscillatoria (Cyanophycées) est le mieux représenté avec 5 espèces suivi du genre Navicula de la classe des Diatomophycées comptant 3 espèces.

Par ailleurs, la diversité spécifique (nombre total d'espèces) au niveau de la Station 1 (32 espèces) est significativement supérieure ( $\mathrm{p}<$
$0,05)$ à celle des Stations 2 et 3 comptant respectivement 13 et 10 espèces.

Il convient de noter que 22 espèces sont spécifiques à la Station 1,4 espèces à la Station 2 et 3 espèces à la Station 3. De plus, les espèces Navicula cryptocephala, Oscillatoria angusta et Tetrasporidium javanicum sont communes aux Stations 1 et 2 tandis que Tetraspora gelatinosa est retrouvée au niveau des Stations 1 et 3. En revanche, Crinalium endophyticum, Eunotia asterionelloides, Nitzschia obtusa, Prasiola velutina, Stigonema minutum, Tabellaria fenestrata sont présentes au niveau de toutes les stations, ce qui témoigne de leur distribution dans tout le collecteur. $\mathrm{La}$ distribution des autres taxa (genre, famille, ordre, classe et embranchement) est similaire à celle des espèces.

\section{Densité phytoplanctonique}

Les densités phytoplanctoniques enregistrées aux différentes stations sont consignées sur la Figure 4. Elles ont varié entre 2870 (Station 2) et 48750 (Station 1) cellules/ml avec une moyenne de $25760 \pm 7348$ cellules $/ \mathrm{ml}$. Aucune différence significative ( $\mathrm{p}$ $>0,05)$ n'a été observée entre les densités phytoplanctoniques obtenues d'une station à une autre.

En outre, Tabellaria fenestra, Nitschia obtusa et Eunotia asterionelloides sont les espèces les plus dominantes.

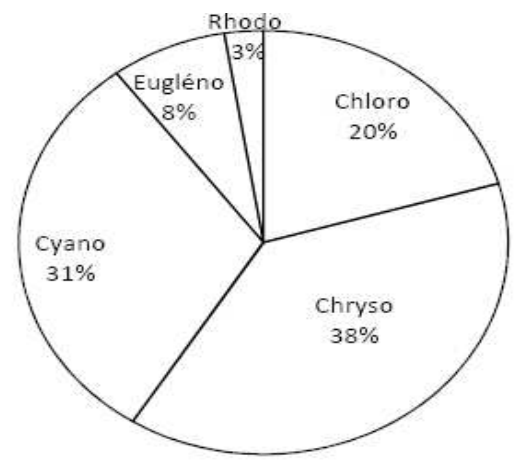

Figure 2: Représentation des embranchements de phytoplancton dans les eaux du collecteur de Dantokpa. Rhodo $=$ Rhodophytes $;$ Chloro $=$ Chlorophytes $;$ Chryso $=$ Chrysophytes $;$ Cyano $=$ Cyanophytes $;$ Eugléno $=$ Euglénophytes. 


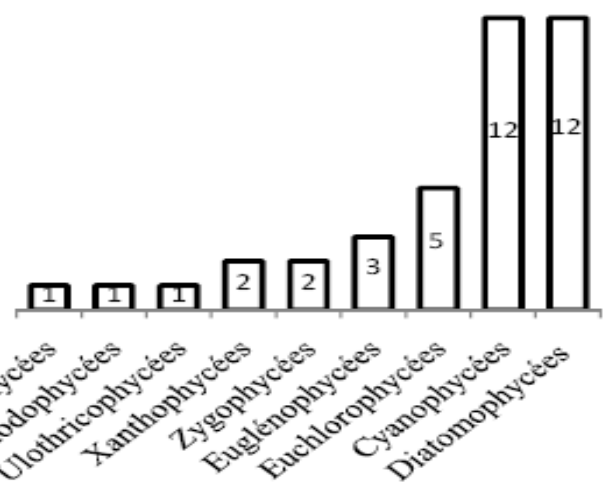

Figure 3: Représentation des classes de phytoplancton dans ces eaux du collecteur de Dantokpa.

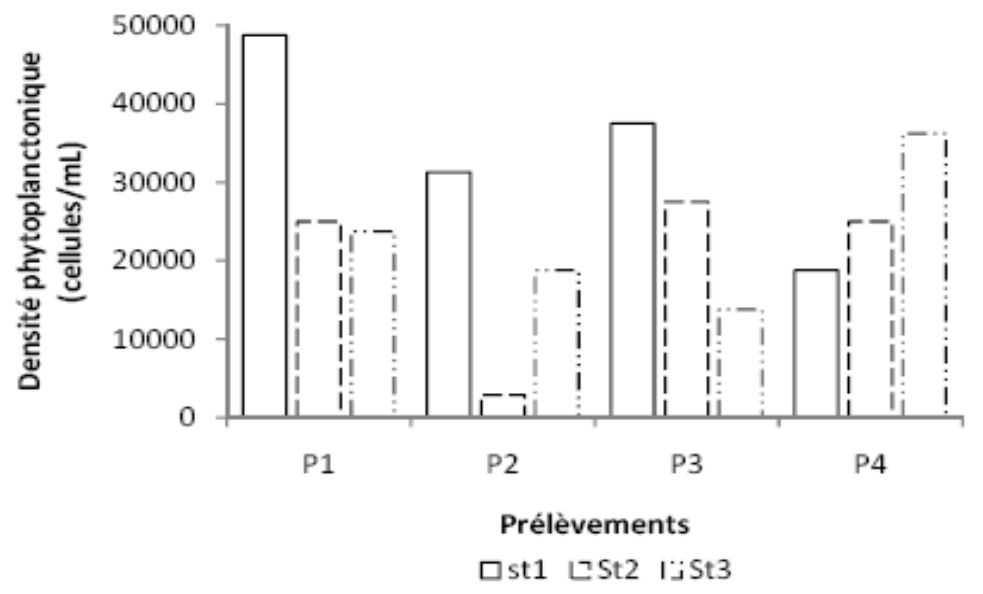

Figure 4: Variations hebdomadaires de la densité phytoplanctonique dans les eaux du collecteur de Dantokpa. $\mathrm{St}=$ Station $; \mathrm{P}=$ prélèvement.

Tableau 1: Moyennes des paramètres physico-chimiques mesurés aux différentes stations dans le canal de Dantokpa.

\begin{tabular}{|c|c|c|c|c|}
\hline \multirow{2}{*}{ Paramètres } & \multicolumn{3}{|c|}{ Moyenne par station \pm Ecart type } & \multirow{2}{*}{$\begin{array}{c}\text { Valeurs } \\
\text { moyennes }\end{array}$} \\
\hline & Station1 & Station2 & Station3 & \\
\hline Température $\left({ }^{\circ} \mathrm{C}\right)$ & $31,57 \pm 2,15$ & $30,22 \pm 1,98$ & $28,80 \pm 1,67$ & $30,20 \pm 1,38$ \\
\hline $\mathrm{pH}$ & $6,51 \pm 0,5$ & $6,84 \pm 0,08$ & $6,75 \pm 0,1$ & $6,70 \pm 0,17$ \\
\hline Turbidité (NTU) & $30,75 \pm 18,19$ & $16,75 \pm 11,35$ & $19,25 \pm 4,50$ & $22,25 \pm 7,46$ \\
\hline $\operatorname{MES}(\mathrm{mg} / \mathrm{l})$ & $25,75 \pm 16,80$ & $12,75 \pm 8,38$ & $15,50 \pm 3,31$ & $18,00 \pm 6,85$ \\
\hline $\mathrm{OD}(\mathrm{mg} / \mathrm{l})$ & $3,37 \pm 0,79$ & $2,82 \pm 0,45$ & $3,22 \pm 0,66$ & $3,14 \pm 0,28$ \\
\hline $\mathrm{NH} 4(\mathrm{mg} / \mathrm{l})$ & $28,28 \pm 7,63$ & $29,40 \pm 26,41$ & $33,94 \pm 10,38$ & $30,54 \pm 2,99$ \\
\hline $\mathrm{NO} 2(\mathrm{mg} / \mathrm{l})$ & $0,21 \pm 0,05$ & $0,50 \pm 0,42$ & $1,58 \pm 1,27$ & $0,76 \pm 0,72$ \\
\hline $\mathrm{NO} 3(\mathrm{mg} / \mathrm{l})$ & $3,20 \pm 1,52$ & $1,83 \pm 0,88$ & $2,35 \pm 0,63$ & $2,46 \pm 0,69$ \\
\hline PO4 (mg/l) & $1,52 \pm 0,65$ & $4,23 \pm 3,81$ & $4,57 \pm 2,78$ & $3,44 \pm 1,67$ \\
\hline $\mathrm{CE}(\mathrm{mS} / \mathrm{cm})$ & $0,74 \pm 0,21^{\mathrm{a}}$ & $0,78 \pm 0,02^{\mathrm{a}}$ & $3,27 \pm 1,13^{\mathrm{b}}$ & - \\
\hline
\end{tabular}


D. C. ADJAHOUINOU et al. / Int. J. Biol. Chem. Sci. 6(5): 1938-1949, 2012

Tableau 2: Répertoire et taxonomie des espèces phytoplanctoniques identifiées dans les eaux du collecteur de Dantokpa.

\begin{tabular}{|c|c|c|c|c|c|}
\hline Classes & Ordres & & Familles & Genres & Espèces \\
\hline \multirow{8}{*}{ Chlorophytes } & Ulothricophycées & Ulvales & Prasiolacées & Prasiola & P. velutina $a^{I ; 2 ; 3}$ \\
\hline & \multirow{5}{*}{ Euchlorophycées } & \multirow[t]{2}{*}{ Chlorococcales } & \multirow{2}{*}{ Oocystacées } & Ankistrodesmus & A. Convolutus ${ }^{I}$ \\
\hline & & & & Kirchneriella & K. elongata ${ }^{I}$ \\
\hline & & \multirow{3}{*}{ Tétrasporales } & \multirow[t]{2}{*}{ Tétrasporacées } & Tetraspora & T. gelatinosa $a^{1 ; 3}$ \\
\hline & & & & Tetrasporidium & T. javanicum ${ }^{1 ; 2}$ \\
\hline & & & Gloéocystacées & Pseudosphaerocystis & P. planctonica ${ }^{I}$ \\
\hline & \multirow[t]{2}{*}{ Zygophycées } & \multirow{2}{*}{ Zygnématales } & Desmidiacées & Pleurotaenium & P. verrucosum ${ }^{I}$ \\
\hline & & & Zygnématacées & Radiofilum & R. transversale ${ }^{3}$ \\
\hline \multirow{11}{*}{ Chysophytes } & Chrysophycées & Phaeoplacales & Sphaeridiothricacées & Stichochrysis & S. immobilis ${ }^{I}$ \\
\hline & \multirow{8}{*}{$\begin{array}{l}\text { Diatomophycées } \\
\text { ou } \\
\text { Bacillariophycées }\end{array}$} & Diatomales & Diatomacées & Tabellaria & T. fenestra ${ }^{1 ; 2 ; 3}$ \\
\hline & & Eunotiales & Eunotiacées & Eunotia & $\begin{array}{l}\text { E. asterionelloides }{ }^{1 ; 2 ; 3} \\
\text { E. parallela }\end{array}$ \\
\hline & & \multirow{5}{*}{ Naviculales } & Naviculacées & Navicula & $\begin{array}{l}\text { N.cryptocephalata }{ }^{1 ; 2} \\
\text { N. } \text { cuspidata }^{l} \\
\text { N. } \text { amphibia }^{l}\end{array}$ \\
\hline & & & \multirow{2}{*}{ Nitzschiacées } & Bacillaria & B. paxilifer ${ }^{3}$ \\
\hline & & & & Nitzschia & $\begin{array}{l}\text { N. pseudobacata }{ }^{2} \\
\text { N. obtusal }{ }^{1 ; 2 ; 3}\end{array}$ \\
\hline & & & Surirellacées & Surirella & S. angustata $^{2}$ \\
\hline & & & Epithémiacées & Rhopalodia & R. $g i b b a^{3}$ \\
\hline & & Rhizosoléniales & Rhizosoléniacées & Rhizosolenia & R. longiseta ${ }^{2}$ \\
\hline & \multirow[t]{2}{*}{ Xanthophycées } & \multirow{2}{*}{ Mischococcales } & Chlorobotrydacées & Heterodesmus & H. multicellularis ${ }^{2}$ \\
\hline & & & Sciadiacées & Ophyocytium & O. parvulum ${ }^{\prime}$ \\
\hline \multirow{3}{*}{$\begin{array}{l}\text { Cyanophytes } \\
\text { ou } \\
\text { Schizophytes }\end{array}$} & \multirow[t]{3}{*}{ Cyanophycées } & \multirow{2}{*}{ Chroococcales } & \multirow{2}{*}{ Chroococcacées } & Tetrarcus & T. ilsteri ${ }^{I}$ \\
\hline & & & & Dzensia & D. salina ${ }^{I}$ \\
\hline & & Nostocales & Nostocacées & Anabaena & A. spiroides ${ }^{I}$ \\
\hline
\end{tabular}


D. C. ADJAHOUINOU et al. / Int. J. Biol. Chem. Sci. 6(5): 1938-1949, 2012

\begin{tabular}{|c|c|c|c|c|c|}
\hline & & & Oscillatoriacées & Oscillatoria & $\begin{array}{l}\text { O. } \text { angusta }^{l ; 2} \\
\text { O. } \text { laxa }^{I} \\
\text { O. subtilissima } \\
\text { O. Schoederi } \\
\text { O. prolifica }\end{array}$ \\
\hline & & & & Lyngbya & L. muralis ${ }^{I}$ \\
\hline & & & & Crinalium & C. endophyticum ${ }^{l ; 2 ; 3}$ \\
\hline & & \multirow[t]{2}{*}{ Stigonématales } & Nostochopsidacées & Baradlaia & B. speluncaecola ${ }^{l}$ \\
\hline & & & Stigonématacées (1) & Stigonema & S. minutum ${ }^{1 ; 2 ; 3}$ \\
\hline \multirow[t]{2}{*}{ Euglénophytes } & \multirow[t]{2}{*}{ Euglénophycées } & \multirow[t]{2}{*}{ Euglénales } & \multirow[t]{2}{*}{ Euglénacées } & Euglena & $\begin{array}{l}\text { E. ehrenbergii }{ }^{I} \\
\text { (var. Africana) } \\
\text { E. ehrenbergii } \\
\text { (sg. Lentiferae) }\end{array}$ \\
\hline & & & & Euglenocaspa & E. ochrace ${ }^{I}$ \\
\hline Rhodophytes & Rhodophycées & Goniotrichales & Goniotrichacées & Kyliniella & K. lavtica ${ }^{I}$ \\
\hline
\end{tabular}

${ }^{1}:$ Station $1 ;{ }^{2}:$ Station $2 ;{ }^{3}:$ Station 3 


\section{DISCUSSION}

Les valeurs moyennes de la température, du $\mathrm{pH}$, des MES sont respectivement égales à $30,2{ }^{\circ} \mathrm{C}, 6,7$ et 18 $\mathrm{mg} / \mathrm{L}$. Elles sont conformes aux normes béninoises de rejet d'eaux résiduaires qui préconisent une température maximale de 30 ${ }^{\circ} \mathrm{C}$, un $\mathrm{pH}$ compris entre 6 et 9 et une quantité de MES inférieure à $35 \mathrm{mg} / \mathrm{l}$.

Le taux moyen d'oxygène dissous égal à $3,14 \mathrm{mg} / 1$ est inférieur à la valeur guide $(6$ $\mathrm{mg} / \mathrm{l}$ ) fixée par l'ordonnance sur le déversement des eaux usées (ODE) et au seuil critique $(4 \mathrm{mg} / \mathrm{l})$ en deçà duquel la vie des microorganismes aérobies est menacée (Cornaz, 2004). Cette faible oxygénation des eaux serait due soit à une consommation importante de l'oxygène dissous par les bactéries aérobies pour la dégradation de la matière organique, soit à une faible activité photosynthétique du phytoplancton (Hamaidi et al., 2009). Elle confère au milieu un caractère anoxique, donc réducteur et asphyxiant. Cet état du milieu justifie d'ailleurs la forte teneur de l'azote ammoniacal $\left(30,54 \mathrm{mg} / \mathrm{l} \mathrm{NH}_{4}{ }^{+}\right)$par rapport à celle des formes oxydées de l'azote $(0,76 \mathrm{mg} / \mathrm{l}$ $\mathrm{NO}_{2}^{-}$et $2,46 \mathrm{mg} / 1 \mathrm{NO}_{3}^{-}$). Notons que le taux moyen d'azote minéral $\left(\mathrm{NH}_{4}{ }^{+}+\mathrm{NO}_{2}^{-}+\mathrm{NO}_{3}{ }^{-}\right)$ estimé à $33,76 \mathrm{mg} / \mathrm{l}$ dans ces eaux est plus de deux fois supérieur à la valeur guide pour l'azote total $(15 \mathrm{mg} / \mathrm{l})$ que préconisent les normes béninoises de rejet des eaux résiduaires.

La concentration moyenne en orthophosphates $(3,44 \mathrm{mg} / \mathrm{l})$, dépasse aussi la valeur fixée par ces normes $(2 \mathrm{mg} / \mathrm{l})$ pour le phosphore total dans les eaux usées domestiques. Cette forte concentration en phosphore dans le canal est due aux eaux usées domestiques et aux urines qui y sont en permanence rejetées par les riverains. En effet, les eaux usées domestiques en raison de leur composition en produits de nettoyage (détergents en l'occurrence) contiennent tout comme les urines, d'importantes quantités de phosphates (Rodier et al., 1996 ; Petry et al., 2002). Elles constituent de ce fait les principales sources de pollution des eaux par les phosphates en milieux urbains (Miquel, 2003).

Les valeurs enregistrées pour la conductivité électrique (CE) indiquent que les eaux du collecteur ont une minéralisation importante en amont (Stations 1 et 2:0,6 ms $\leq \mathrm{CE} \leq 1 \mathrm{~ms}$ ) et excessive (Station 3: $\mathrm{CE}>1$ ms) en aval (Genoud et al., 1997). Ceci témoigne d'une forte teneur en sels dissous confirmant ainsi les concentrations élevées des minéraux azotés et phosphatés dans le canal.

Le phytoplancton tout comme les macrophytes, les invertébrés et les poissons sont des bio-indicateurs c'est-à-dire qu'ils donnent une indication sur la qualité de leur biotope de part la diversité de leur taxon, leur assemblage, la présence ou l'absence de groupes sensibles aux pollutions (Rolland, 2009).

$\begin{array}{cccc}\text { L'analyse } & \text { de } & \text { la } & \text { composition } \\ \text { taxonomique } & \text { du } & & \text { peuplement }\end{array}$ phytoplanctonique place en tête les Cyanophycées et les Diatomophycées. La forte proportion des cyanophycées se justifie par maints facteurs du milieu dont :

- la forte disponibilité du phosphore sous forme d'orthophosphates pour lesquels les cyanobactéries présentent plus d'affinité que les autres algues (Blais, 2002).

- la faible oxygénation et la forte turbidité, conditions généralement défavorables à la prolifération algale mais dans lesquelles les cyanobactéries survivent (Lavoie et al., 2007), ce qui les rend plus compétitifs.

De plus, parmi les cyanophycées identifiées, les espèces des genres Anabaena, Lyngbya et Oscillatoria sont très compétitives grâce à leur capacité à secréter des cyanotoxines de différents types (Affsa/Afsset, 2006) qui inhibent la croissance des autres groupes d'algues et éliminent leurs prédateurs comme le zooplancton (Lavoie et 
al., 2007). Par ailleurs, les espèces des genres Anabaena, Crinalium, Lyngbya, Oscillatoria, appartenant à l'ordre des Nostocales et celles des genres Baradlaia, Stigonema de l'ordre des Stigonématales sont pourvues d'hétérocyste, structure qui leur permet de fixer l'azote atmosphérique. Cette propriété les rend très compétitives par rapport aux autres algues lorsque l'azote est limitant (Blais, 2002).

En ce qui concerne les Diatomophycées, leur forte proportion notée dans le peuplement phytoplanctonique des eaux analysées est bien en accord avec les résultats trouvés par Zerhouni (2003) dans les eaux usées de la ville de Fès (Maroc).

La proportion non moins importante des Euchlorophycées est due au pH acide des eaux qui favorise leur croissance (Reyssac, 1970).

La faible proportion des Chrysophycées et des Rhodophycées justifie le caractère eutrophe des eaux analysées. En effet, ces groupes de phytoplancton prolifèrent dans les milieux aquatiques oligotrophes où les eaux sont pures (Nicholls, 1995). Par ailleurs, cet état d'eutrophisation est corroborée non seulement par la forte densité phytoplanctonique moyennement évaluée à 25760 cellules/ml mais aussi par la présence des genres Anabaena (A. spiroides) caractéristiques des eaux riches en éléments nutritifs et Nitzschia (N. pseudobacata et $N$. obtusa) présentant leur optimum de croissance dans les eaux fortement eutrophisées (Descy et Willems, 1991).

En outre, la forte représentation du genre Oscillatoria (5 espèces), bon indicateur d'une forte concentration en matière organique (Chopin, 2001), et la présence des deux espèces de l'embranchement des Euglénophytes caractéristiques des milieux riches en substances organiques (Iltis, 1980), confirment une fois encore l'importance des charges organiques dans notre milieu d'étude.

La similarité des données physicochimiques enregistrées dans la présente étude serait due au fait que cette dernière ait été conduite dans une période située entièrement dans la saison sèche. Cela n'a donc pas permis d'apprécier la dynamique spatio-temporelle du phytoplancton qui est entre autres contrôlée par la variation des paramètres physico-chimiques (Komoé et al., 2009). L'analyse des différents paramètres physicochimiques étudiés en particulier l'oxygène dissous, la conductivité électrique et les concentrations en minéraux azotés et phosphatés ainsi que celle de la composition taxonomique du phytoplancton a néanmoins permis d'indiquer la forte charge minérale et organique dans les eaux du collecteur. Ces données s'accordent par conséquent sur la nécessité de traiter ces eaux avant leur rejet dans le milieu naturel. Leur déversement sans traitement, comme c'est le cas actuellement, participerait donc à l'eutrophisation graduelle du milieu aquatique récepteur avec pour corollaire la baisse de sa biodiversité et son comblement à long terme. Aussi, la présence des espèces de cyanobactéries potentiellement toxiques pose-t-elle un problème de santé publique. Il est établi que leurs cyanotoxines (hépatotoxines, neurotoxines, dermatotoxines) sont très dangereux pour les animaux et en dernier ressort pour l'homme (Vonarx, 2008).

\section{Conclusion}

Les résultats issus de cette étude signalent à travers la diversité taxonomique du phytoplancton et les paramètres physicochimiques, l'état de pollution très avancée des eaux du collecteur. Ils montrent par conséquent l'importance des charges polluantes organiques et minérales déversées dans la lagune de Cotonou via le présent collecteur et sans nul doute les nombreux autres érigés dans la ville. Il urge donc de penser sérieusement à l'assainissement des eaux usées de la ville et d'entreprendre leur épuration avant leur déversement dans les milieux aquatiques afin de conserver d'une part la biodiversité de ces derniers et d'autre 
part, la santé des populations qui s'en servent pour l'alimentation, la boisson, la navigation, l'habitat....

Ce travail confirme par ailleurs tout l'intérêt que revêt la systématique des algues dans l'appréciation de la qualité de l'eau. Aussi, a-t-il permis de faire un premier inventaire des espèces phytoplanctoniques caractéristiques de ces écosystèmes particuliers. Des études ultérieures étendues sur un cycle annuel devront apprécier la dynamique du phytoplancton et entreprendre une caractérisation complète des eaux usées de la ville de Cotonou en vue de poser des bases solides pour la maîtrise de leur épuration.

\section{REMERCIEMENTS}

Nous exprimons notre gratitude au Ministère de l'Enseignement Supérieur et de la Recherche Scientifique du Bénin qui ont entièrement financé ces travaux de recherches.

\section{REFERENCES}

Adam KS, Boko M. 1993. Le Bénin (collection $\mathrm{n}^{\circ} 26,5^{\mathrm{eme}}$ edn). Edicef : France; p. 95.

Afssa/Afsset, 2006. Évaluation des risques liés à la présence de cyanobactéries et de leurs toxines dans les eaux destinées à l'alimentation, à la baignade et autres activités récréatives. Rapport commun sur les risques sanitaires liés à la présence de cyanobactéries dans l'eau, p. 232.

Blais S. 2002. La problématique des cyanobactéries (algues bleu-vert) à la baie Missisquoi en 2001. Agrosol., 13(2): 103110.

Bourrelly P. 1981. Les Algues d'Eau Douce: Algues Jaunes et Brunes (Tome 2). Edition Boubée N. et Cie: Paris; p.521.

Bourrelly P. 1985. Les Algues d'Eau Douce: Algues Bleues et Rouges (Tome 3). Edition Boubée N. et Cie: Paris; p. 608.
Bourrelly P. 1990. Les Algues d'Eau Douce: Algues Vertes (Tome 1). Edition Boubée N. et Cie: Paris; p. 576.

Chopin T. 2001. Protocole pour la surveillance des algues marines. Environnement Canada, p. 36. www.ec.gc.ca consulté le 13/02/2012.

Compère P. 1974. Algues de la région du lac Tchad. II- Cyanophycées. Cah. O.R.S.T.O.M., ser. Hydrobiol., 8(3/4): 165-198.

Compère P. 1975 a. Algues de la région du lac Tchad. III- Rhodophycées, Euglénophycées, Cryptophycées, Dinophycées, Chrysophycées, Xanthophycées. Cah. O.R.S.T.O.M., ser. Hydrobiol., 9(3): 167-192.

Compère P. 1975 b. Algues de la région du lac Tchad. IV- Diatomophycées. Cah. O.R.S.T.O.M., ser. Hydrobiol., 9(4): 203290.

Compère P. 1976 a. Algues de la région du lac Tchad. V- Chorophycophytes $1^{\text {ere }}$ partie. Cah. O.R.S.T.O.M., ser. Hydrobiol., 10(2): 77-118.

Compère P. 1976 b. Algues de la région du lac Tchad. VI- Chorophycophytes $2^{\mathrm{e}}$ partie: Ulotrichophycées, Zygnématacées. Cah. O.R.S.T.O.M., ser. Hydrobiol., 10(3): 135-164.

Compère P. 1977. Algues de la région du lac Tchad. VII- Chorophycophytes $3^{\mathrm{e}}$ partie : Desrnidiées. Cah. O.R.S.T.O.M., ser. Hydrobiol., 11(2): 77-177.

Cornaz S. 2004. Evaluation du statut trophique d'un canal de drainage sous l'impact des pollutions d'origines diffuses et ponctuelles, le cas du grand canal de plaine de Rhône. Institut de Géographie de l'Université de Lausanne, p.180.

Descy JP, Willems C. 1991. Contribution à la connaissance du phytoplancton de la Moselle (France). Cryptogamie, Algologie, 12(2): 87-100.

Dovonou EF. 2008. La pollution des plans d'eau au Benin. Mémoire de DEA/Centre 
Inter-facultaire pour la Formation et la Recherche en Environnement et Développement (CIFRED), p. 67.

Effebi R. 2009. Lagunage anaérobie : modélisation combinant la décantation primaire et la dégradation anaérobie. Thèse de doctorat, Université de Liège Campus d'ARLON, p. 235.

Findlay DL, Kling HJ. 2003. Protocoles de mesure de la biodiversité : le phytoplancton d'eau douce. Institut des Eaux douces. 501 University Crescent Winnipeg (Manitoba) R3T 2N6, p.21.

Genoud L, Bagnoud R, Baudin MN, Furrer P, Venetz PM. 1997. Etude du réseau de drainage de la plaine de Vionnaz. Service de la Protection de L'Environnement et Laboratoire Cantonal du Canton du Valais, p. 85.

Hach. 2004. DR/890 Datalogging colorimeter handbook. Hach company world headquarters, Loveland, Colorado, p.616.

Hamaidi MS, Hamaidi F, Zoubiri A, Benouaklil F, Dhan Y. 2009. Etude de la dynamique des populations phytoplanctoniques et résultats préliminaires sur les blooms toxiques a cyanobacteries dans le barrage de Ghrib (Ain Defla-Algérie). European Journal of Scientific Research, 32(3): 369-380.

Helm MM, Bourne N, Lovatelli A. 2006. Ecloserie de bivalves. Un manuel pratique. Document technique sur les pêches. No. 471. Rome, FAO. p. 184.

Iltis A. 1980. Les algues. In Flore et Faune Aquatiques de l'Afrique SahéloSoudanienne (Tome I), Durand JR, Lévêque C (eds). Éditions ORSTOM, Collection Initiations Doc. Tech. 44: Paris; 9-61.

Komoé K, Da Kouhété P, Kouassi AM, Aka NM, Kamanzi AK, Ama AA. 2009. Seasonal Distribution of Phytoplankton in Grand-Lahou Lagoon (Côte d'Ivoire). European Journal of Scientific Research, 26(3): 329-334.
Koné D. 2002. Epuration des eaux usées par lagunage à microphytes et à macrophytes en Afrique de l'ouest et du centre: état des lieux, performances épuratoires et critères de dimensionnement. Thèse de Doctorat ès Sciences Techniques, Faculté Environnement Naturel, Architectural Et Construit /Ecole Polytechnique Fédérale de Lausanne, $\mathrm{N}^{\circ}$ 2653, p.170.

Lavoie I, Laurion I, Warren A, Vincent WF. 2007. Les fleurs d'eau de Cyanobactéries. Revue littéraire. INRS rapport $\mathrm{n}^{\circ} 916$, xiii, p.12.

Miquel G. 2003. La qualité de l'eau et de l'assainissement en France. Office Parlementaire d'Evaluation des Choix Scientifiques et Technologiques (Sénat), Rapport n²15, Tome 1, p.195.

MMEE. 2007. Ministère des Mines, de l'Energie et de l'Eau/Bénin. Stratégie nationale de l'assainissement des eaux usées en milieu urbain 2008-2015, p.34. www.rncrbenin.org consulté le 05/08/11.

Morel M, Kane M. 1998. Le lagunage à Macrophytes, une technique permettant l'épuration des eaux usées pour son recyclage et de multiples valorisations de la biomasse. Sud Sciences et Technologies, 1: 5-16.

Nicholls KH. 1995. Chrysophytes bloom in the plankton and neuston of marine and freshwater systems. In Chrysophytes Algae: Ecology, Phylogeny and Development, Sandgren CD, Smol JP, Kristiansen J (eds). Cambridge University Press: UK.; 181-216.

Petry J, Soulsby C, Malcolm IA, Youngson AF. 2002. Hydrological controls on nutrient concentrations and fluxes in agricultural catchments. The Science of the Total Environment, 294: 95-110.

Reyssac O. 1970. Phytoplancton et production primaire au large de la Côte-d'Ivoire. Bul. IFAN, Sér. Arch., 32(4): 869-981.

Rodier J, Bazin C, Broutin JC, Chambon P, Champsaur H, Rodi L. 1996. L'Analyse 
de l'Eau ( $8^{\text {ème }}$ édn). Dunod: Paris; p. 1383.

Rolland A. 2009. Dynamique et diversité du phytoplancton dans le réservoir Marne (bassin versant de la seine). Thèse de Doctorat/ Université de Savoie/Ecole Doctorale SISEO, p.242.

Seidl M, Mouchel JM. 2003. Valorisation des eaux usées par lagunage dans les pays en voie de développement. Centre d'Enseignement et de Recherche Eau Ville Environnement, p.43.

UADE, OIEAU. 1993. Gestion des déchets liquides et solides dans les centres urbains d'Afrique. Ministère Français de la coopération, Séminaire Assainissement Urbain en Afrique, Rapport final du Séminaire Assainissement Urbain en Afrique, Ministère Français de la coopération.
Vonarx J. 2008. Cyanobactéries et cyanotoxines. p.12. www.snv.jussieu.fr consulté le 09/02/11.

Zerhouni RA. 2003. Flore algale des eaux usées de la ville de Fès et étude de la capacité de certaines espèces à éliminer la charge azotée, phosphatée et quelques métaux lourds (Chrome et Cadmium). Thèse de Doctorat/ Université Sidi Mohamed Ben Abdellah/Faculté des Sciences Dhar el Mahraz /Fès (Maroc), p. 154 .

Zerhouni RA, Bouya D, Ronneau C, CARA J. 2004. Etude de l'absorption des phosphates, azote, chrome et cadmium par trois algues vertes isolées à partir d'effluents urbains. Rev. Sci. Eau, 17(3): 317-328. 